\title{
An Analysis of MLRS Near-Real-Time Earth Orientation Results
}

\author{
Randall L. Ricklefs and Peter J. Shelus \\ McDonald Observatory and Department of Astronomy \\ University of Texas at Austin \\ Austin, Texas 78712-1083 \\ USA
}

\begin{abstract}
In this paper a comparison is presented between near-real-time earth orientation parameters, produced on-site by the McDonald Laser Ranging System (MLRS) at McDonald Observatory, using observations to the Apollo 15 lunar retroreflector, and those results which are obtained after the fact at the University of Texas at Austin and elsewhere, as well as the results obtained from other techniques. The MLRS data set which is included in this study spans the interval from the commencement of on-site earth orientation solutions at MLRS in February 1985, through the present time, September 1986. This research is supported by the National Aeronautics and Space Administration under Grant NAG5-754 and Contract NAS5-29404 to McDonald Observatory and the University of Texas at Austin from the Goddard Space Flight Center in Greenbelt, Maryland.
\end{abstract}

\section{INTRODUCTION}

The securing of precise Earth orientation information has been the one of the major goals of laser ranging observations to Earth orbiting targets for quite a long time (see, for example, Bender et al, 1973 or Mulholland, 1980). Although much successful work has been performed at various analysis centers over the past ten years in producing these results, spurred on by such international projects as EROLD and MERIT (see, for example, Langley et al, 1981 or Dickey and Williams, 1983), the presence of powerful mini- and micro-computers on-site at modern laser ranging stations, coupled with the long period modeling accuracies now available from the analysis of those observations, make it possible for one to consider the computation of these data products in a near-realtime environment on-site at the laser ranging stations themselves. At the McDonald Observatory Laser Ranging Station (MLRS), located near Fort Davis, Texas, we have been producing such results, using lunar laser ranging observations, since early 1985 . This paper describes the techniques which are being applied and the results which have so far been obtained.

\section{TECHNIQUES}

The basic equation for dynamical parameter improvement which is used in most lunar laser ranging analyses is the following: 


$$
\begin{array}{cc}
n & \partial \tau \\
\tau_{\mathrm{o}}-\tau_{\mathrm{c}}=\sum_{\mathrm{i}=1} & \partial \kappa_{\mathrm{i}}
\end{array}
$$

where $\tau_{\mathrm{o}}$ and $\tau_{\mathrm{c}}$ are the observed and calculated ranges (i.e., time delays), respectively, and the $\kappa_{\mathrm{i}}$ are parameters associated with the model currently being used. Since it is convenient to deal with changes in the Earth's orientation as being represented by changes in the longitude, $\lambda$, and the latitude, $\phi$, of the observing station, one can re-write equation (1) explicitly as (see, for example, Stolz and Larden, 1977 or Shelus et al, 1981):

$\tau_{\mathrm{o}}-\tau_{\mathrm{c}}=\mathrm{r}[\cos \phi \cos \delta \sin \mathrm{H}] \Delta \lambda+\mathrm{r}[\sin (\phi-\delta)-\sin \phi \cos \delta(1-\cos \mathrm{H})] \Delta \phi+\mathrm{C} .(2)$

$\mathrm{H}$ is the local lunar hour angle, $\delta$ is the lunar declination, $\mathrm{r}$ is the radial distance, and $\mathrm{C}$ is a constant. For present purposes, it has been assumed that all short period terms having amplitudes of $1 \mathrm{~cm}$ or more, other than those dealing with the orientation of the earth, have been eliminated in the modeling and that, since we shall be averaging data over no more than a few days, all longer period terms can be assumed to be constant. We feel that this is certainly a reasonable assumption for the lunar system.

Of course, the major requirement for the production of real-time earth orientation information on-site (in addition to the capability of making the observations themselves) is the availability of a convenient and precise lunar range prediction system. The transfer of such a system to the MLRS was accomplished two years ago when we succeeded in reproducing MIT lunar range prediction results at the $20-30$ psec level. We are presently working to attain the same levels of accuracy with the JPL lunar model predictions.

The obtaining of earth orientation parameters from MLRS ranging observations can be summarized as follows. With the completion of a lunar "run", using various interactive graphics tools, the lunar data set is statistically filtered and mathematically compressed to a single normal point. When several such normal points have been formed within a pre-defined interval of time (usually 24-48 hours), an earth orientation solution is performed using the residual and partial derivative values appropriate to equation 2 above. At the present time, only observations to the Apollo 15 (Hadley) retroreflector are used and only a longitude, i.e., UT- 0 , solution is made. We consider an earth orientation reduction to be "prime" if it is obtained using data within a single lunar transit and with an hour angle spread of at least 3 hours. Otherwise, the reduction is considered to be "nonprime". Results are inspected on-site for potential problems and, if satisfactory, are placed onto the General Electric Mark III system for distribution. Normally, the total time, from the beginning of the observing sequences for a "prime" data set to Earth orientation parameter transmittal, encompasses an interval of less than 18 hours.

\section{RESULTS}

Since February, 1985 there have been some 38 UT-0 points produced at the MLRS in this real-time environment. Of these, 8 are considered "prime". Comparisons of these "quick-look" results with "full-rate" results obtained by X X Newhall at JPL (Figure 1), those obtained by the BIH (Figure 4), and those obtained in Austin (Figures 2 and 5) are quite satisfactory. For the JPL comparisons, it was necessary to convert the "standard" UT0 $0_{R}$ - UTC values to UT0 - UTC by re-introducing the short period zonal tide terms 
(see Yoder et al, 1981). For the BIH comparisons, UT0 - UTC values at McDonald Observatory were inferred from the BIH final values for $x, y$, and UT1 - UTC, interpolated to the time of the MLRS UT-0 point using a 4-point interpolation scheme. As a consistency check for our analysis, other UT-0 values, derived with the same software from University of Hawaii (HOLLAS) lunar ranging data, are also compared with values from JPL (Figure 3) and BIH (Figure 6).

As can be seen from the graphs, there are two outlying points in Figure 4 which do not appear in the other graphs. The actual ranging data which had been used to produce those points were later shown to be of marginal quality and have since been eliminated from the total MLRS data set. The outlying point in both of the final MLRS comparisons (Figures 2 and 5) near MJD 46200 is a two-day ("non-prime") point with a single lunar run being used from each day. Similarly, the three outlyers from the HOLLAS-BIH comparisons (Figure 6) are also two-day points. More work is needed to identify the reasons why two-day points usually compare poorly with one-day points. Perhaps weak data or poor calibrations is the reason. At the present time, and until a satisfactory explanation is in hand, all "non-prime" points will be explicitly identified in the data formats accompanying our Earth orientation data. Further, the logic of some of this software and some of the data filtering techniques have been evolving to select only the very strongest data for use in this "quick-look" earth orientation analysis.

It should be noted that the earth orientation results from MLRS (Figures 1,2,4, and 5) and HOLLAS (Figures 3 and 6) were all obtained with the same software and each shows a definite positive bias away from zero. If one takes the unweighted mean and standard deviation of these offsets after removing obvious outlyers (see Table I) one also sees a slight bias. The fact that this bias exists is most probably due to the use of the MIT ephemeris for our predictions. We plan to test this hypothesis when the JPL ephemeris/libration package has been implemented for MLRS. Also, the simple fact that an average difference of 0.31 ms exists between the BIH and JPL offsets, if that is significant, may indicate a systematic difference in processing. JPL tentatively reports values $0.633 \mathrm{~ms}$ higher than BIH (Newhall, et al, 1986).

Table I. Comparison of UTO biases using MLRS reduction system

\begin{tabular}{lcc}
\hline Differenced Values & $\begin{array}{c}\text { Bias } \\
(\mathrm{ms})\end{array}$ & $\begin{array}{c}\text { Standard Deviation } \\
(\mathrm{ms})\end{array}$ \\
\hline MLRS-JPL (quick-look): & 1.25 & 1.02 \\
MLRS-JPL (final): & 1.58 & 0.78 \\
MLRS-BIH (quick-look): & 1.42 & 1.39 \\
MLRS-BIH (final): & 1.88 & 0.93 \\
HOLLAS-JPL: & 1.37 & 1.00 \\
HOLLAS-BIH: & 1.84 & 0.89 \\
\hline
\end{tabular}

As a caveat to prospective users of these "quick-turnaround" results, it is quite important to realize that this is essentially a "quick-look" procedure. Since we wish to deal in a real-time environment, the results can sometimes be contaminated by data which later proves to be poor, under further analysis. Also, at the MLRS there is no fitting of the data for other parameter improvement, as is done in the regular after-the-fact, full-rate 


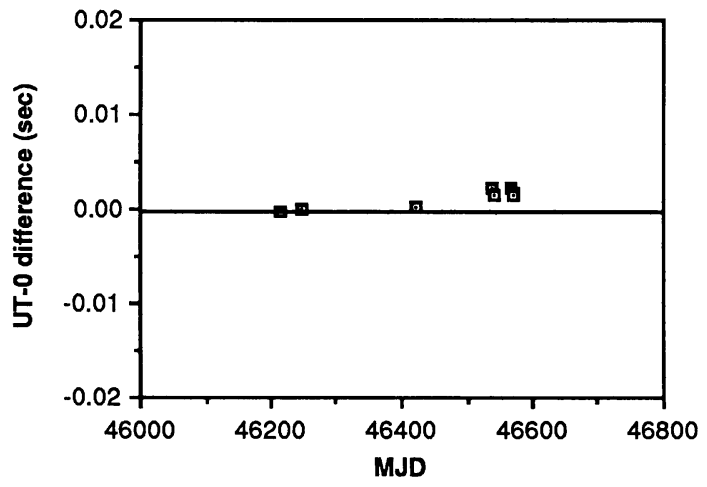

Figure 1. MLRS-JPL (quick-look)

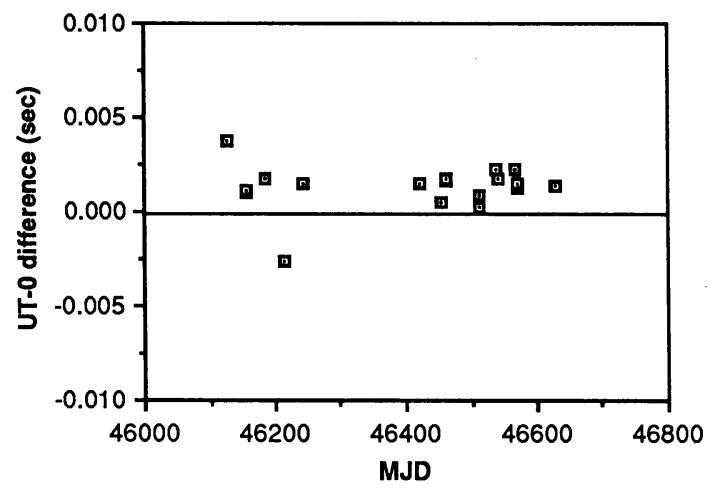

Figure 2. MLRS-JPL (final)

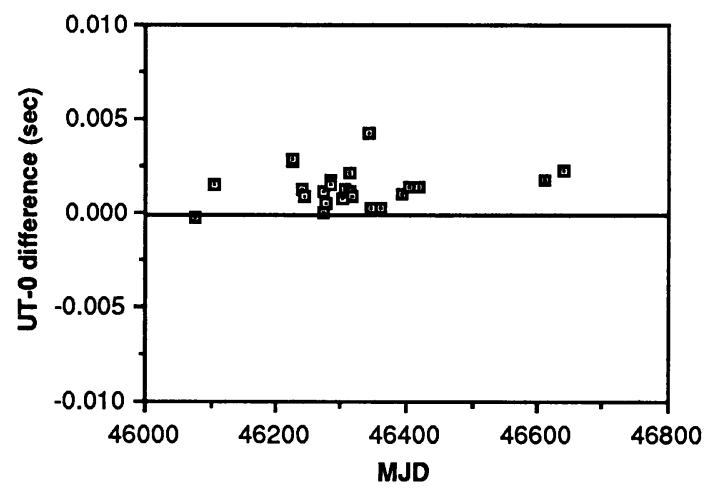

Figure 3. HOLLAS-JPL 


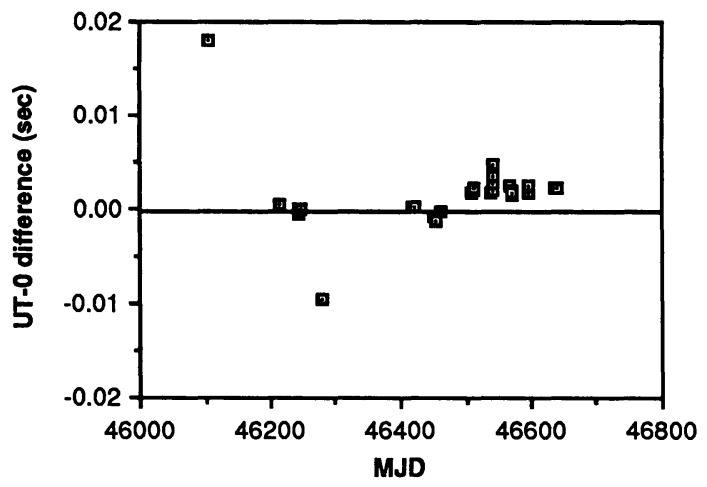

Figure 4. MLRS-BIH (quick-look)

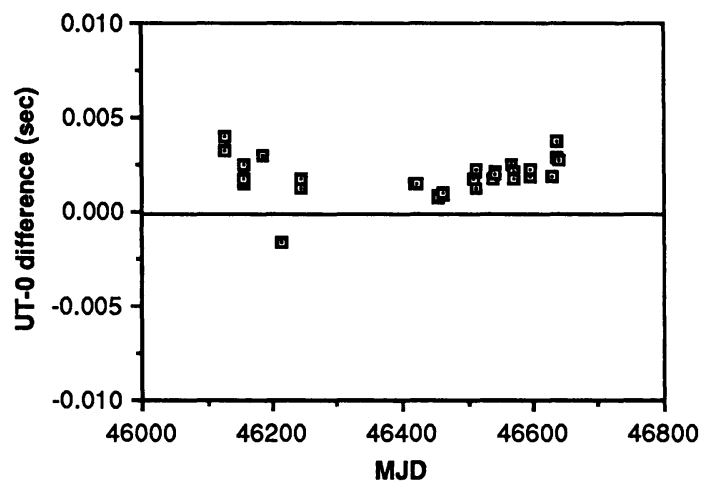

Figure 5. MLRS-BIH (final)

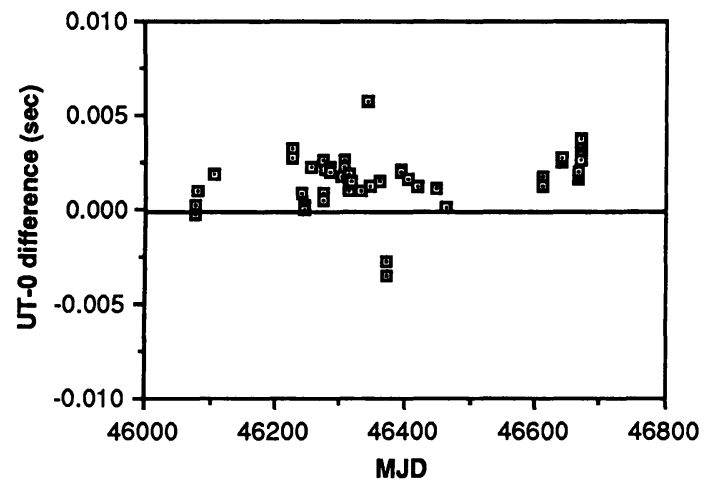

Figure 6. HOLLAS-BIH 
data environment. In spite of these shortcomings, we feel that the product is a good one and can serve many of the "quick-look" needs of the Earth orientation community.

As a final comment, it must be stated that the biggest problem which confronts us now is a paucity of Earth orientation results using this lunar technique. At the MLRS, the station has been working flawlessly; the data gaps are completely a product of the environment. It is hoped that most of this type of problem can be solved by a move of the MLRS from its present "saddle-site" to one at the top of a near-by mountain. On the positive side, in our processing of lunar laser ranging data from the Haleakala site, we are attempting to apply the self-same MLRS data reduction techniques to compute Earth orientation parameters from Hawaii data. In addition, we are also interested in the sharing this software with other stations, and are encouraging them to undertake similar "quick-look" types of analyses to obtain additional earth orientation results.

\section{ACKNOWLEDGEMENTS}

The authors cannot too strongly thank Robert W. King at MIT for his invaluable aid in the implementation of the MIT lunar range prediction package at the MLRS. Additional computer equipment, which provides the capability of simultaneous data gathering and data reduction at the MLRS, has been secured, in part, through funding provided by Dennis D. McCarthy of the U. S. Naval Observatory. Many thanks also go to Nelson Zarate, who produced large parts of the original version of the Earth orientation analysis routines, and to Janice Storm, who runs the present software system so productively at the MLRS. We also thank X X Newhall at JPL for feedback on MLRS UTOR-UTC values and the crew at HOLLAS, especially Lou Macknik and Dan O'Gara for the Maui lunar data. Finally, we acknowledge that this research is being supported at McDonald Observatory and the University of Texas at Austin by National Aeronautics and Space Administration Grant NAG5-754 AND Contract NAS5-29404 of Goddard Space Flight Center, Technical Officers Dr. Gilbert D. Mead and Mr. Robert L. Appler, respectively.

\section{REFERENCES}

Bender, P.L., Currie, D.G., Dicke, R.H., Eckhardt, D.H., Faller, J.E., Kaula, W.M., Mulholland, J.D., Plotkin, H.H., Poultney, S.K., Silverberg, E.C., Wilkinson, D.T., Williams, J.G., and Alley, C.O. 1973, 'The Lunar Laser Ranging Experiment', Science, 182, 229-238.

Dickey, J.O. and Williams, J.G. 1983, 'Earth Rotation from Lunar Laser Ranging', Astron. Astrophy. Suppl. Ser., 54, 519-540.

Langley, R.B., King, R.W., and Shapiro, I.I. 1981, 'Earth Rotation from Lunar Laser Ranging', J. Geophys. Res., 86, 11913-11918.

Mulholland, J.D. 1980, 'Scientific Achievement from Ten Years of Lunar Laser Ranging', Rev. of Geophy. and Sp. Phys., 18, 549-564.

Newhall, X X, Dickey, J. O., and Williams, J. G. 1986, 'Comparison of LLR UT1 with. IRIS UT1', JPL Engineering Memorandum EM 335-71.

Shelus, P.J., Zarate, N.R., and Eanes, R.J. 1981, 'Earth Rotation from a Simultaneous Reduction of LLR and LAGEOS Laser Ranging Data', Proceedings of the 63rd Coll. of the IAU, O. Calame, ed., D. Reidel, 31-40.

Stolz, A. and Larden, D. 1977, 'Accuracy Obtainable for Universal Time and Polar Motion During the EROLD Campaign', Scientific Applications of Lunar Laser Ranging, J.D. Mulholland, ed., D. Reidel, 37-50.

Yoder, C.F., Williams, J.G., Parke, M.E., and Dickey, J.O. 1981, 'Short Period Tidal Variations of Earth Rotation', Ann. des Geophy., 37, 213. 